systematic reviews meeting all inclusion criteria. Of the 24 identified reviews, 16 reviews addressed question 1, whilst 19 reviews addressed question 2 . The primary studies within these reviews included a number of interventional studies and several randomised control trials.

The majority of studies which examined the optimum age of transition stated that transition time should be determined by developmental maturity, not a rigid age threshold. However, delayed transition was associated with improved outcomes. These included health-service use, clinical outcomes, reduced care gaps and patient satisfaction. Evidence from individual programmes found potential benefits from commencing transition preparedness training in early adolescence.

Studies which evaluated age-appropriate services found that they were generally well-regarded by patients and often result in improved outcomes, better engagement with healthcare services or improved patient health behaviours.

Conclusions This review suggests that delayed transition and dedicated age-appropriate services result in improved outcomes and increased patient satisfaction. These findings appear to be consistent across a range of long-term conditions and in a variety of healthcare systems. Further work is required to identify specific barriers and facilitators to successful healthcare transition.

\section{G425(P) A SYSTEMATIC REVIEW AND META-ANALYSIS OF OUT OF HOSPITAL NURSING INTERVENTIONS TO REDUCE EMERGENCY DEPARTMENT ATTENDANCES IN CHILDREN AND YOUNG PEOPLE}

${ }^{1} \mathrm{R}$ Satherley, ${ }^{2} \mathrm{~K}$ Lonergan, ${ }^{3} \mathrm{D}$ Devakumar, ${ }^{3} \mathrm{R}$ Cheung, ${ }^{3} \mathrm{M}$ Cortina-Borja, ${ }^{4} \mathrm{M}$ Heys, ${ }^{1} \mathrm{I}$ Wolfe. ${ }^{1}$ School of Population Sciences and Health Services Research, King's College London, London, UK; 'Division of Health and Social Care Research, King's College London, London, UK; ${ }^{3}$ Institute of Child Health, University College London, London, UK; ${ }^{4}$ Evelina London Children's Hospital, Guy's and St Thomas', London, UK

\subsection{6/archdischild-2018-rcpch.414}

Aims Rising use of emergency departments has resulted in increased costs and poor quality of care for children and young people. Clear evidence on which interventions work in reducing the number of unplanned admissions is important for those who use and commission emergency department services and to improve the quality of healthcare services. There is a policy imperative to shift care out of hospitals but insufficient evidence on the effectiveness of out of hospital care. This review aims to identify, critique, and collate outcomes in published evidence for nurse-led out hospital care for children and young people with chronic conditions.

Methods Two databases were systematically searched from 1965-2017, to identify randomised controlled trials that used nurse-led out of hospital care to reduce emergency department attendances in children and young people (0-18 years) with at least one chronic condition. The pooled incidence rate ratio (IRR) was estimated using the $\mathrm{R}$ package metaphor.

Results Five randomised controlled trials (3 USA, 1 Canada, 1 Scotland) met the inclusion criteria. All five trials were included in the qualitative review but four were included in the meta-analysis due to heterogeneity in outcome measures. Four papers reported on CYP with asthma and the fifth on chronic illness. Only three papers reported significant effect for a reduction in emergency department attendances. Study quality was moderate, with a medium risk of bias. The meta- analysis fitted a random-effects model, which estimated a pooled IRR of 0.65 (95\% CI $0.40,1.03)$ implying a non-significant positive effect of nurse-led out of hospital care on reducing emergency department attendance.

Conclusions Although this review found no association between emergency department usage and nurse-led out of hospital care, the effect sizes were large and three papers found positive associations. Only five randomised controlled trials were included in this review, most of which studied children and young people with asthma, highlighting the need for further research in this area.

\section{G426(P) PAEDIATRIC DENTISTS' IDENTIFICATION AND MANAGEMENT OF UNDERWEIGHT AND OVERWEIGHT CHILDREN}

${ }^{1}$ EM Clark, 'D Tuthill, ${ }^{2} E J$ Hingston. 'Paediatrics and Child Health, Noah's Ark Children's Hospital for Wales, Cardiff, UK; ${ }^{2}$ Paediatric Dentistry, University Dental Hospital, Cardiff, UK

\subsection{6/archdischild-2018-rcpch.415}

Aim Dental caries and obesity are increasing challenges for the NHS. Dentists see children frequently and are well placed to identify both conditions and potentially intervene, in line with government strategy of 'making every contact count'. We aimed to identify routine practice of Specialists in Paediatric Dentistry (SPD) regarding diagnosis and management of children with abnormal body mass index (BMI).

Methods An anonymous online survey was emailed to all SPD in the UK. Questions investigated whether and when height, weight or BMI were measured; actions taken; and dentists' feelings regarding their role.

Results 49/112 (42\%) of SPDs responded (table 1). All felt they had a responsibility to identify underweight or overweight/obese children.

Abstract G426(P) Table 1 Dentists' habits regarding assessment of children's weight, height, and BMI

\begin{tabular}{|c|c|c|c|c|c|c|}
\hline $\begin{array}{l}\text { How frequently do you measure } \\
X \text { ? }\end{array}$ & $\begin{array}{l}\text { Weight } \\
\mathrm{n}\end{array}$ & $\%$ & $\begin{array}{l}\text { Height } \\
\mathrm{n}\end{array}$ & $\%$ & $\begin{array}{l}\text { BMI } \\
\mathbf{n}\end{array}$ & $\%$ \\
\hline Always & 8 & 17 & 7 & 15 & 3 & 7 \\
\hline Often & 12 & 26 & 11 & 24 & 9 & 20 \\
\hline Occasionally & 18 & 39 & 14 & 30 & 13 & 28 \\
\hline Rarely & 2 & 4 & 3 & 7 & 4 & 9 \\
\hline Never & 6 & 13 & 11 & 24 & 17 & 37 \\
\hline
\end{tabular}

Weight was the most commonly measured anthropometric value; height less so. BMI was most commonly calculated if a general anaesthetic/sedation was planned $(n=21 / 29)$, or if the patient 'looked overweight' $(n=16 / 29)$. Of those measuring BMI only $41 \%$ had taken action more than twice in a year: most commonly (90\%) the child's GP was informed. Challenges to intervention included the sensitive nature of weight, and the lack of protocol. Of the $37 \%$ that did not measure BMI, two-thirds felt they did not know how to interpret the result.

Conclusions SPD were supportive of their role in the identification of underweight or overweight/obese children presenting to their clinics. However, many felt uncertain about BMI 